\title{
LASERS AND PHOTOPOLYMERS
}

\author{
C. CARRE, C. DECKER, J. P. FOUASSIER and D. J. LOUGNOT \\ Laboratoire de Photochimie Générale, URA 431 du CNRS, \\ Ecole Nationale Supérieure de Chimie, 3 rue A. Werner, 68093 Mulhouse \\ Cedex, France.
}

(Received 20 November 1989; in final form 15 January 1990)

\begin{abstract}
A general outline of new applications of lasers in polymer photochemistry is given. The fields covered include the kinetic and mechanistic study of various elementary processes (in solution model compounds) by laser spectroscopies and the extension of this approach to practical systems (solids or in polymerization samples, thin films) by holographic techniques or real-time infrared spectroscopy. Stress is laid on the great importance of laser techniques in understanding the fundamental aspects of the processes involved in polymer photochemistry.
\end{abstract}

KEY WORDS: Photoinitiation, photopolymerization, laser spectroscopy, holography, infrared spectroscopy, excited states.

\section{INTRODUCTION}

The applications of lasers in polymer photochemistry spring from the meeting point of two fascinating research fields. First, light-curable monomers are increasingly developed, due to the rapid growth of the industrial applications in various areas, such as adhesives, dental and medical equipment, inks, metal decoration, flooring, coatings and varnishes (optical fibers, wood, plastics, papers . . .), printing plates, printed circuits. Improvement of the systems requires further investigation on the (photo)-physical and (photo)-chemical processes involved (in particular, the photoreactivity of sensitizers used to start the reaction) and new ideas concerned with the use of powerful irradiation devices in the case of specific applications. Second, during the past decade laser sources appeared as very convenient tools in photochemistry and photophysics. One of their most attractive applications lies in using a short pulse of laser light as an excitation source to generate transient states whose absorption is monitored, in real time, with a colorimetric analysis device. This technique has been successfully applied to the study of a large variety of processes in molecules ${ }^{1}$ and macromolecules. ${ }^{2}$ Other emerging applications range in the field of laser-induced chemistry and holographic recording in polymer systems. The present paper deals with both aspects and stresses the fact that lasers used as irradiation sources and probes can improve the knowledge of the primary processes involved in the excited states of polymerization photoinitiators and the development of new applications. 


\section{OUTLINE OF THEORY}

Photopolymerization of unsaturated multifunctional monomers (according to a radical process) and, to a lesser extent, cyclic monomers (through a ring opening cationic reaction) is widely used..$^{3-4}$ Direct absorption of light by monomers being a rather inefficient process, sensitive organic molecules are added to start rapidly the reaction: a photoinitiator $\mathrm{I}$ is able to generate directly reactive species $R^{\bullet}$ or $C^{\oplus}$ through light excitation, whereas a photosensitizer $S$ absorbs light and transfers its excitation to a photoinitiator. The advantage of using a photosensitizer is to absorb light at higher wavelengths (and to recover photons that are generally lost) or/and to match the absorption with a given wavelength of the emitting light source (e.g., when using laser light beams). ${ }^{5}$ In a general way, ${ }^{6}$ when UV light excitation takes place, the photoinitiator is promoted to its first excited singlet state, which then converts into its triplet state, via fast intersystem crossing. Generally, this transient state yields reactive radical or ionic species which are capable of attacking a monomer molecule (M) and to initiate the polymerization. Unfortunately, the triplet transient state can be deactivated through several processes: quenching by oxygen, non radiative or radiative processes, formation of complexes and photolysis products, quenching by the monomer through process which does not involve any chain initiation (accordingly, this process must be regarded as a dead loss pathway). The role of the photoinitiator is of prime importance with regard to the efficiency of the initiation process, and it is worthy of note that the process involved in the excited states governs what is called "reactivity." Very powerful techniques for investigating these processes are based on time resolved laser spectroscopy devices, which makes it possible to excite, in a very short time, a population of molecules and to monitor, in real time, the relaxation or the evolution of the excited molecules or fragments and, in this way, to determine the rate constants of the various reactions involved.

\section{TIME-RESOLVED ABSORPTION LASER-SPECTROSCOPY OF EXCITED STATE PROCESSES}

A typical device of laser spectroscopy with a nanosecond time resolution is shown in Figure $1 .^{7}$ The excitation source consists of a Neodynium-YAG Q-switched by a Pockels cell which produces a $3 \mathrm{~ns}$ pulse at $1,06 \mathrm{~nm}, 532 \mathrm{~nm}, 355 \mathrm{~nm}$ or $265 \mathrm{~nm}$. The monitoring system consists of a Xenon arc, a monochromator, a photomultiplier at the detection end, a transient digitizing system and a computer station. In some cases, when the wavelengths of the excitation pulses which are directly available from the $N d / Y A G$ lasers are not adapted to the requirements of a selective excitation, a laser-pumped-dye-laser is used. The secondary laser emission obtained from the dye serves as a new excitation source of the molecules studied; its time profile is similar to that of the pump laser.

\section{Investigation of Primary Processes}

Time-resolved laser spectroscopy gives a better insight into the dynamics of the excited states and provides a valuable description of the processes involved, which 


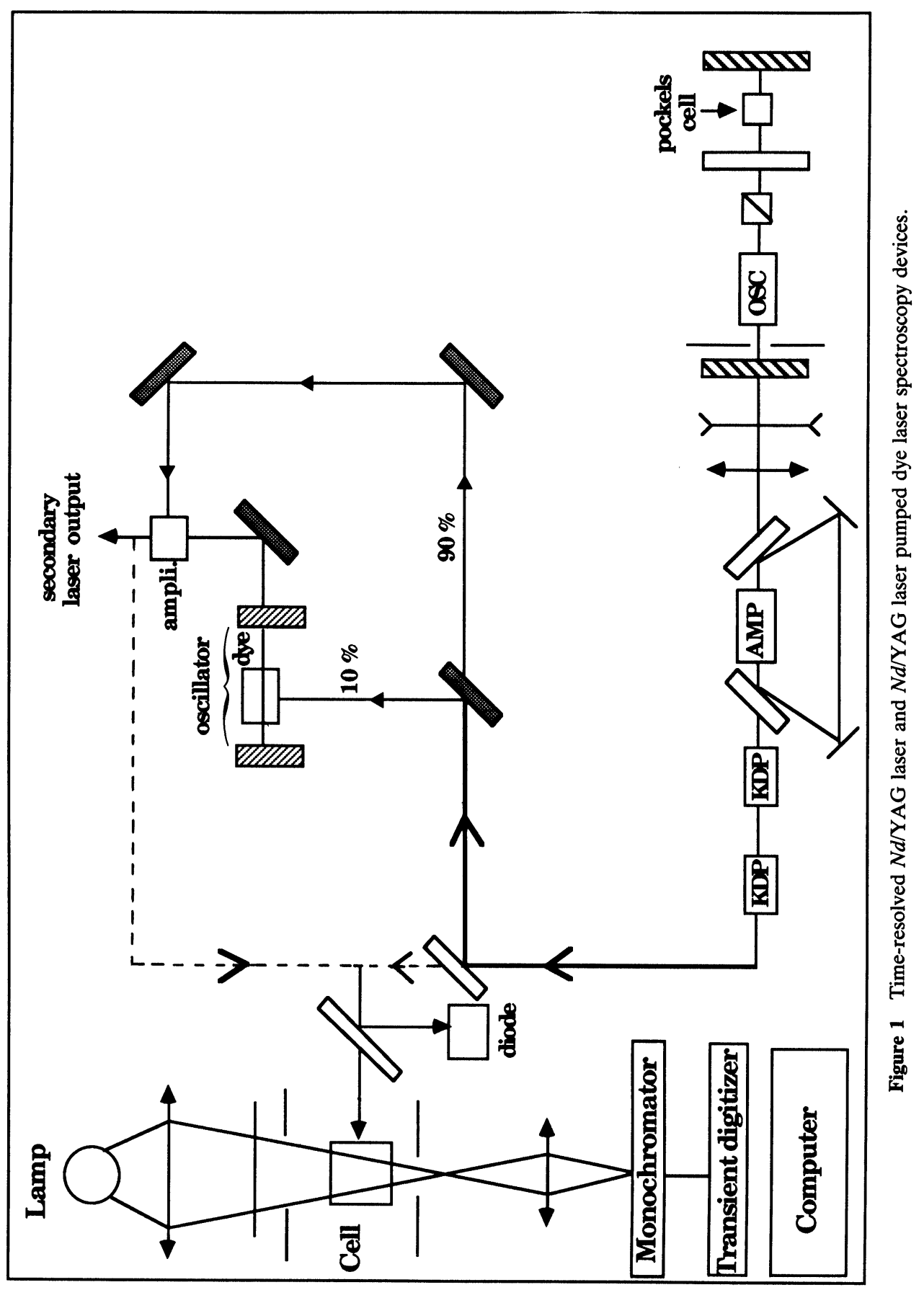


may be helpful to explain the relative efficiency of photoinitiators and to inquire into possible improvements of the reactivity of various molecular structures. This experimental probing into the molecular reactivity makes it possible to ask one fundamental question: how can the most efficient photoinitiators be selected? This is an important question both for the organic chemists, who want to synthesize the most beneficial molecules, and the industrial users who generally demand photosensitive systems with the highest rate of polymerization $R p$. At the present time, a lot of rate constants corresponding to cleavage $k_{\alpha}$ or/and $e^{-}$transfer $k_{e}$, monomer (M) quenching $k_{q}$ have been measured. ${ }^{8-22}$ The related quantum yield $\Phi_{\alpha}$ and $\Phi_{C T}$ can be computed. By putting these values together with those of the rates of polymerization obtained in well defined conditions, one is able to calculate the quantum yield of radical attack on the monomer unit $\Phi_{R M}$, e.g:

with

$$
\text { photoinitiator } \stackrel{\Phi_{\alpha}}{\longrightarrow} R^{\bullet} \stackrel{\Phi_{R M}}{\longrightarrow} R M^{\bullet}
$$

$$
R_{p}=K \sqrt{\Phi_{\alpha} \Phi_{R M}} \text { and } \Phi_{\alpha}=\frac{k_{\alpha}}{k_{\alpha}+k_{q}[M]}
$$

Complete diagrams of evolution of the excited states after light excitation have been proposed for a large variety of ketones used as photoinitiators e.g., phenyl acetophenones, ${ }^{8}$ benzophenones, ${ }^{9}$ dialkoxy acetophenones, ${ }^{10}$ sulfonyl ketones, ${ }^{11}$ water-soluble ketones, ${ }^{12}$ hydroxy alkyl ketones, ${ }^{13}$ acyl phosphine oxides ${ }^{14}$ Specific effects on the reactivity-efficiency relationships have been investigated: substituent and structural effects, ${ }^{15}$ environment ${ }^{16}$ monomer quenching, electron transfer in the presence of amines, reactivity of the radicals towards the double bond. ${ }^{17}$

In a similar manner, an investigation of the primary processes in cationic photopolymerization could be very helpful in elucidating the mechanism of the initiation. Recent research-work was carried out on iodonium salts. Progress has been achieved concerning the mechanism of the spectral sensitization from the excited states of the donor. ${ }^{18-19}$ Much has to be done in the understanding of the processes involved in onium salts ${ }^{20}$ and organo metallic compounds. ${ }^{21}$

Visible photosensitizers have always been considered as attractive either for direct initiation through visible light (conventional or laser beams) or to increase the energy dose received by a substrate exposed to a polychromatic light. This can be achieved by using substituted photoinitiators exhibiting a red shifted absorption, ketocoumarins, organo metallics, dyes. Sensitization of radical or cationic polymerization can be obtained in the presence of these compounds. A full knowledge of the mechanisms of electron and energy transfer, ${ }^{22}$ as investigated through laser spectroscopy, will help to the design of more efficient and well adapted photosensitive systems working under the constraining conditions imposed by exposure to laser beams.

\section{Excitation Transfer in Ketones as studied by Laser Pumped Dye Laser Spectroscopy}

Very recently, it was shown that mixing substituted thioxanthones e.g., TX with the morpholino ketone MK extends the photosensitivity towards the near visible part of 
the spectrum and accelerates the curing of pigmented coatings. ${ }^{23}$ Investigating directly the processes involved between TX and MK during the exchange of excitation energy requires a pumping pulsed source of light being able to excite TX without exciting MK: this is readily achieved by using a light beam delivered by a dye laser pumped with a Nd-YAG laser. ${ }^{7}$

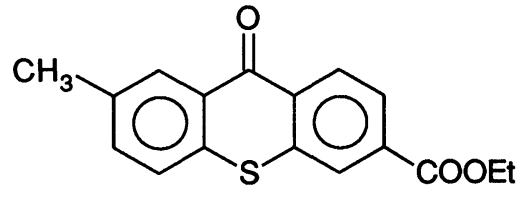

TX

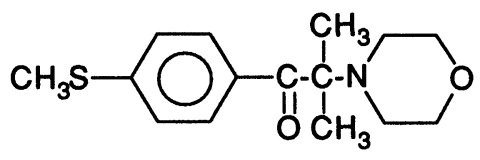

MK

Fluorescence and triplet absorption are quenched when methyldiethanolamine (MDEA) is added to a solution of TX and ketyl species are formed. In the case of $\mathrm{MK}$, a fast cleavage occurs in a very short lived triplet state $(\leqslant 10 \mathrm{~ns})$ generating a methylthiobenzoyl-morpholino isopropyl radical pair that undergoes disproportionation or separation. The absorption of the methylthio benzoyl radical is observed as well as the generation of a ketyl structure through a quenching of the triplet state by amines. The usual bimolecular quenching of triplet states by monomer is also observed.

The interactions between TX and MK was investigated through laser pumped dye laser spectroscopy at $\lambda=430 \mathrm{~nm}$ and steady state fluorescence measurements. This interaction is drastically affected by a solvent effect: when going from toluene to methanol, the quenching rate constant of the triplet state increases by almost an order of magnitude whereas the ketyl radical absorption is only detected when the experiment is carried out in toluene. These results reveal that excitation transfer occurs mostly through energy transfer in methanol (and in polar solvents) and electron transfer in toluene (and in non polar solvents). The relative efficiencies of these two competitive processes (Figure 2) are dependent of the stabilization of the lowest excited state $T_{1}$ by solvatation (that governs the spectroscopic character of $T_{1}$ ) and on the relative positions of the triplet energy levels of the donor TX and the acceptor MK (which is deciding for the possibility of an energy transfer process).

\section{HOLOGRAPHIC SPECTROSCOPY}

During recent years, new experimental techniques in which information is gained from the study of the temporal behavior of optical gratings have drawn the attention of physical chemists. Their outstanding and really seductive features are related to many potential applications as non destructive tools for the study of physical or chemical transformations occurring in particular media where the conventional methods of investigation cannot be resorted to. In a simplified manner, two different types of techniques ${ }^{24-25}$ based on the use of holographic gratings can be distinguished. The experiments in which the kinetics of building up and/or fading of a photochemically induced grating is studied, and referred to as holographic spectro- 


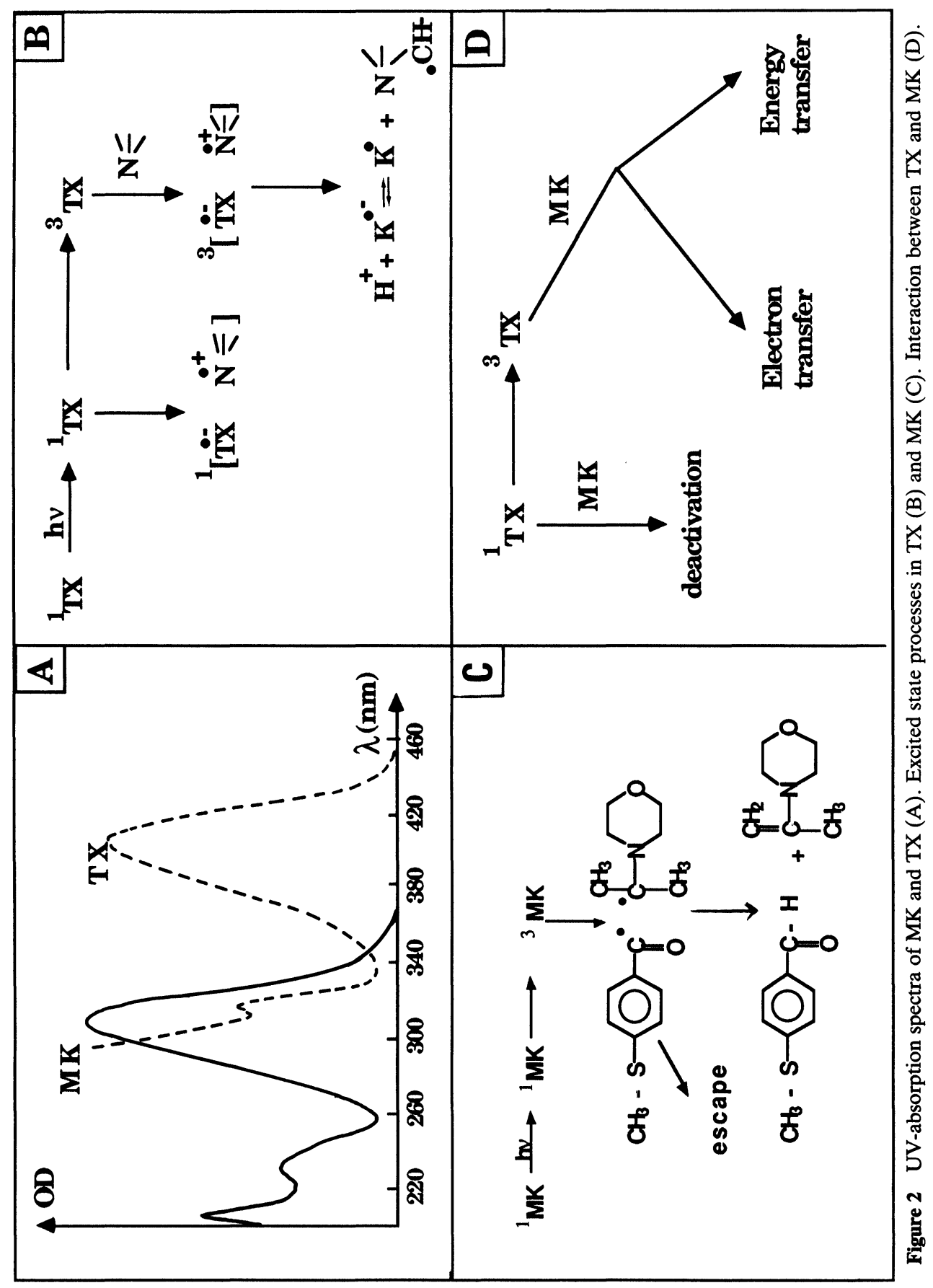


scopy experiments; those in which a diffracted beam generated by an hologram memorizing the initial stage of a photochemically sensitive system, interferes with a beam transmitted or reflected by this system at any stage of its transformation, are known as holographic interferometry experiments.

When polymerizable thin films are illuminated by an interference pattern, photopolymerization (or any other photoprocess) takes place only in the bright areas of this pattern. If the problem of the response of the material is considered from a purely qualitative point of view, one can intuitively expect a degree of phototransformation imaging the spatial distribution of the incident light intensity, i.e., a "counterpart" of the interference pattern. This spatially modulated degree of transformation is thus associated with the creation in the photosensitive medium of a volume or amplitude hologram. ${ }^{26-34}$ Three types of modulation can be involved: a depth modulation resulting from an inhomogeneous shrinkage effect, a modulation of the refractive index which is a consequence of the heterogeneous distribution of segment density or chain length and finally, a spatial modulation of the absorbency of the material going along with the inhomogeneous bleaching of ground state species. In this context, several authors have introduced theoretical analysis of the correlation between the spatial structure of the incident pattern, the response of the material (in terms of holographic efficiency) and the time of illumination. ${ }^{26}$ These approaches which use simple analytic expressions derived from the coupled wave theory developed by Kogelnik in the case of thick sinusoidal gratings, lead to satisfactory analysis provided the underlying assumptions are satisfied. ${ }^{35-36}$ Several examples are reported in the literature, in which the time evolution of the holographic efficiency curves related to a one step photoreaction is shown to be a quadratic function of the irradiation time. However, after the very first moments of a photoreaction in a polymer material, this simplified approach is often inadequate. This is due to the fact that, in most of these photosensitive systems, the rate of polymerization is not proportional to the incident intensity ${ }^{37}$ Thus, the refractive index modulation induced by the interference of two sinusoidal plane waves cannot be assumed to exhibit a sinusoidal profile. We have recently introduced a theoretical statement of this question, the conclusions of which are quite in favor of the application of holographic techniques to many physiochemical studies devoted to polymerizable systems. ${ }^{38} \mathrm{Up}$ to now, they have been used with profit for kinetic and mechanistic investigations of the polymerization process (by monitoring the building up of the diffraction efficiency/time curves). They are also well adapted to kinetic studies of the processes contributing to the bleaching of an holographic record "written" in a polymeric material (by monitoring the decay of these curves).

A typical experimental set up used in this kind of study is shown in Figure 3. The hologram is created with a continuous wave "writing" laser, the output of which is split into two beams of equal intensity. So as to get rid of problems of coherence length, a system of adjustable mirrors allows the beams to interfere with equal pathlengths. The full angle of interference $2 \theta$ (typically $25^{\circ}$ ) controls the fringe spacing $\Lambda(1,42 \mu \mathrm{m})$. This laser beam is expanded so as to allow efficient spatial filtering. Doing so, the incident interference pattern can be considered to be due to the mixing of two coherent plane waves. When dealing with volume phase holo- 


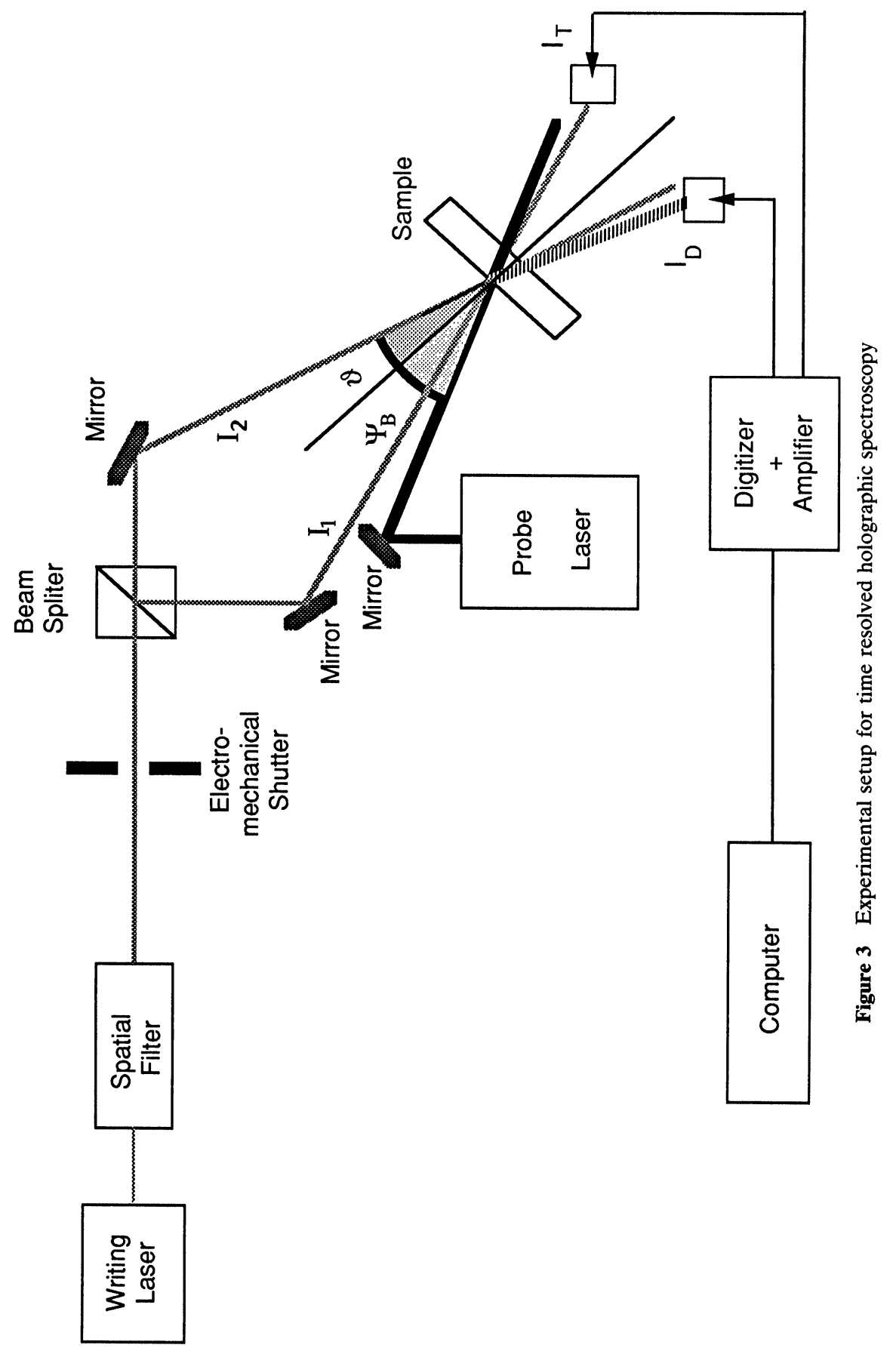


grams, the record is generally "read" in continuous with an other laser, the wavelength of which falls into a spectral "window" of the reactive mixture (i.e. a region where it is completely transparent). The interest of this procedure lies in the fact that the probe laser beam is inactive toward the recording material. Thus, it becomes possible to follow the building up of the hologram as a function of the irradiation time without pertubating the refractive index modulation. The diffraction efficiency $\eta$ which is defined as the ratio of the intensity diffracted by the grating to the incident intensity is measured in continuous by a system of photovoltaic cells. The same holds true with systems in which the relaxation of an hologram is monitored. In this case, an electromechanical shutter placed in the path of the writing laser, with a $1 \mathrm{~ms}$ commutation time, controls the time resolution of the system. Two detectors interfaced with a computer measure the intensity of the diffracted beam and the transmittance of the sample, i.e., the averáge concentration of the absorbing species (photoinitiator or sensitizer).

\section{Comparative Reactivity of Amines as Coinitiators for Acrylic Radical Polymerization}

When dealing with comparative reactivity studies on acrylic radical polymerization. macromolecular chemists are often faced with the non-trivial question of the intrinsic reactivity of the coinitiator in the initiator/coinitiator systems. This initiator works according to the following reaction scheme:

$$
\begin{aligned}
& \text { initiator } \stackrel{\text { light }}{\longrightarrow} \text { initiator* } \\
& \text { initiator* }+ \text { coinitiator } \longrightarrow \text { initiator }{ }^{\bullet}+\text { coinitiator }^{\bullet} \\
& \text { coinitiator }^{\bullet} \stackrel{\text { 'monomer }}{k_{i}} \text { polymer chain }
\end{aligned}
$$

Whatever its structure, the oxydo-reduction process which affects the excited initiator lends itself to thorough kinetic and spectroscopic studies by laser spectroscopy. On the opposite, the behavior of coinitiator-derived radicals is rather difficult to investigate owing to their transparency in the spectral range covered by timeresolved techniques. As a consequence, the rate constant of initiation $\left(k_{i}\right)$ or the corresponding quantum yield cannot be determined directly. Moreover when dealing with practical polymerizable formulations which are used in photocuring technologies, the material in its final form does not lend itself to simultaneous rate $\left(R_{p}\right)$ and degree (DP) of polymerization measurements, hence the impossibility to deduce any information concerning the parameter $k_{i}$. We have recently developed an original approach of this question which is based on the simultaneous study of the kinetic of building up of a volume phase hologram in the photosensitive material and of the progressive bleaching of the initiator as the polymerization progresses. ${ }^{38}$ Thus, in such systems, the existence of a linear relationship between the instantaneous diffraction efficiency of the grating $\eta(t)$ and $B(t)$ has been demonstrated (Figure 4):

$$
\eta(t)=\alpha B(t)+\beta \quad \text { with } \quad \alpha=\frac{K \sqrt{\Phi}}{\Phi_{T}}
$$

(where $K$ is an experimental constant which is related to the chemistry (rate constant of the redox process) and to the set up (incident intensity); $\Phi_{T}$ is the quantum yield of the process generating the excited state precursor; $\Phi$ (overall initiation quantum 


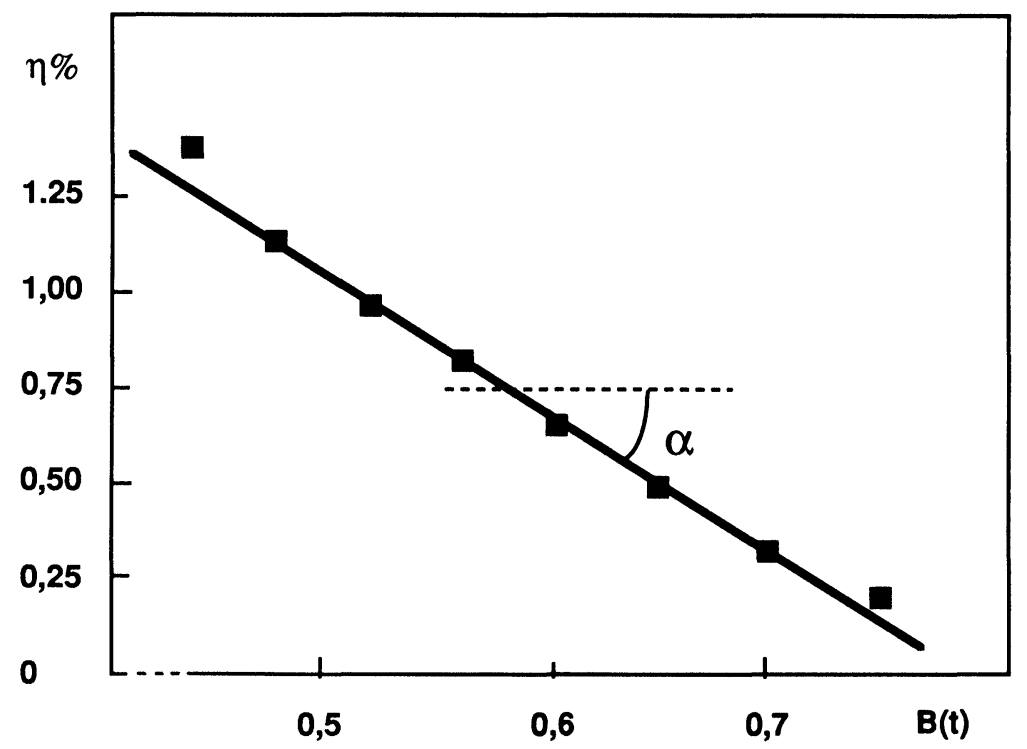

Figure 4 Linear correlation between $\eta \%(t)$ and $B(t)$ in the system Acrylamide/Methylene Blue $\left[5 \times 10^{-3}\right.$ M]/Diethanolamine [1M].

yield) $\left.=\Phi_{T} \Phi_{R} \Phi_{i}\right)$ and

$$
\begin{gathered}
B(t)=\int_{\mathrm{OD}_{0}}^{\mathrm{OD}_{t}} \frac{d(\mathrm{OD})}{\left(1-10^{-\mathrm{OD}}\right)^{1 / 2}} \\
(\mathrm{OD}=\text { Optical density of the sample) }
\end{gathered}
$$

This overall yield $\Phi$ contains $\Phi_{i}$ which is nothing else that the quantum efficiency of the process leading to effective chain initiation. Since $\Phi_{T}$ and $\Phi_{R}$ can be determined by other techniques (sensitization, laser spectroscopy), the relative value of $\Phi_{i}$ in a series of synergists exhibiting basically the same photochemical behaviour becomes available. For example, Table 1 summarizes a few results obtained in acrylamide polymerization in the presence of the Methylene Blue/amine system. Thus, by applying this new approach to polymerizable systems, it becomes possible to appreciate the relative part played by the two consecutive steps of the overall initiation mechanism: the photochemically induced production of the initiating radical and its reaction with monomer molecules.

Table 1 Experimental results (TEA is taken as an internal reference to improve legibility).

\begin{tabular}{llllc}
\hline Amine & $\Phi_{T} \Phi_{R}$ & $-\alpha$ & $\Phi_{\mathrm{i}}$ & $\eta_{\max }(\%)$ \\
\hline Triethanolamine (TEA) & 1.0 & 1.0 & 1.0 & 2.0 \\
Diethanolamine (DEA) & 1.5 & 0.78 & 0.52 & 1.8 \\
Ethanolamine (EA) & 1.9 & 1.79 & 0.94 & 1.6 \\
Dimethylbenzylamine (DMBA) & 2.9 & 0 & 0 & 0 \\
Butoxyethyl dimethylaminobenzoate (BEA) & 0.5 & 0.005 & 0.01 & 10 \\
\hline
\end{tabular}

$\left(\lambda_{\text {writing }}=633 \mathrm{~nm}(\mathrm{He}-\mathrm{Ne}\right.$ laser $)$ and $\mu_{\text {probe }}=488 \mathrm{~nm}\left(\mathrm{Ar}^{+}\right.$laser $)$. Film thickness $\left.=80 \mu \mathrm{m} ; 2 \vartheta=25^{\circ}\right)$. 


\section{Polymer Matrix Effects on the Kinetics of Chemical Reactions}

A great number of chemical processes (either thermal or photochemical) with which the photochemists have a fair acquaintance and those consequences are perfectly controlled in solution, often remain difficult to elucidate in polymers. Moreover, when such reactions take place in rigid matrixes which due to their shape (thin films or weakly transparent samples) do not lend themselves to classical spectroscopic investigations, any kinetic analysis becomes quite problematical. In this context, time resolved holographic spectroscopy (TRHS) appears in some specific cases as a very powerful tool for their study. By way of illustration, a recent study of the matrix effects on the cis-trans isomerization of azo compounds (such as methyl orange) exemplifies its usefulness in this field. The photoprocess affecting the light fastness of this class of dyes are rather well known in solution and the mechanism of their isomerization and photobleaching has been the object of many investigations, the conclusions of which are now reputedly self-consistent. However, the concepts which prevail in solution cannot be merely transposed to polymer systems. Going from a solution to a polymer matrix is expected to hugely reduce the rate of the processes involving a large twist of the molecule; moreover, the thermal recovery of the trans form from the metastable cis isomer is known to be catalyzed by protons, ${ }^{39} \mathrm{a}$ process which must be affected by the solid polymer matrix:
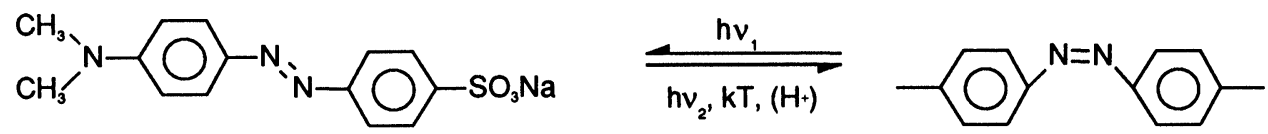

The interest of using holographic spectroscopy in this kind of system is twofold: first, this zero background technique allows the study of thin films in which the very weak changes of absorptivity going along with the cis-trans isomerization are far below the sensitivity limit of time-resolved laser spectroscopy: second, since the information is associated with a volume phase hologram, the sample can be interrogated with any laser wavelength falling out of the absorption spectrum of the species involved. The spurious effects induced by the analysis beam can be completely eliminated. Thus, the recovery times deduced from a holographic spectroscopy investigation can be regarded as much more accurate values than the ones measured by any other time resolved technique. A few interesting and meaningful results referring to Methyl Orange dissolved in polyvinyl alcohol ${ }^{40}$ are reported in Figures 5, 6,7 and Table 2. A typical curve $\eta=f(t)$ is reported in Figure 5 for a given incident intensity $I$. As seen on the insert, the time-dependence of $\sqrt{\eta}$ is linear, with a slope $k$ during the first moments of the photoisomerization (as long as the absorption regime remains independent on the absorbed dose). A linear correlation (Figure 6) is obtained when $\log k$ is plotted against $\log I$ with a slope $\alpha \approx 1$. This result leads to the conclusion that the number of photon mechanistically involved in the process is close to one. i.e. the trans-cis isomerization is monophotonic in this particular medium. Moreover, the study of the relaxation of the transient grating associated to the cis isomer by TRHS (Figure 7) leads to a lifetime (whatever the incident dose used to create the holographic record): $\tau \approx 5 \mathrm{~s}$ 


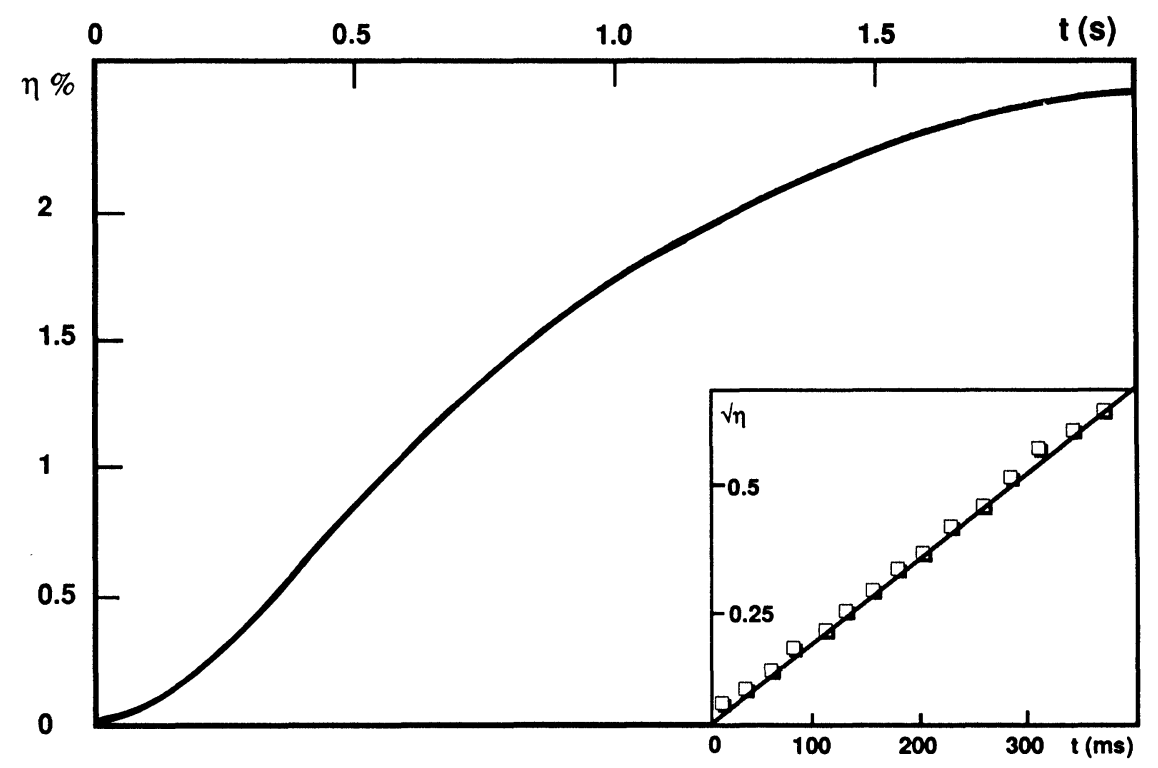

Figure 5 Building up of the hologram recorded with Methyl Orange in PVA. The inset corresponds to the curve $\sqrt{\eta}=f(t) ; I=3.5 \mathrm{~mW}-\mathrm{cm}^{2}$.

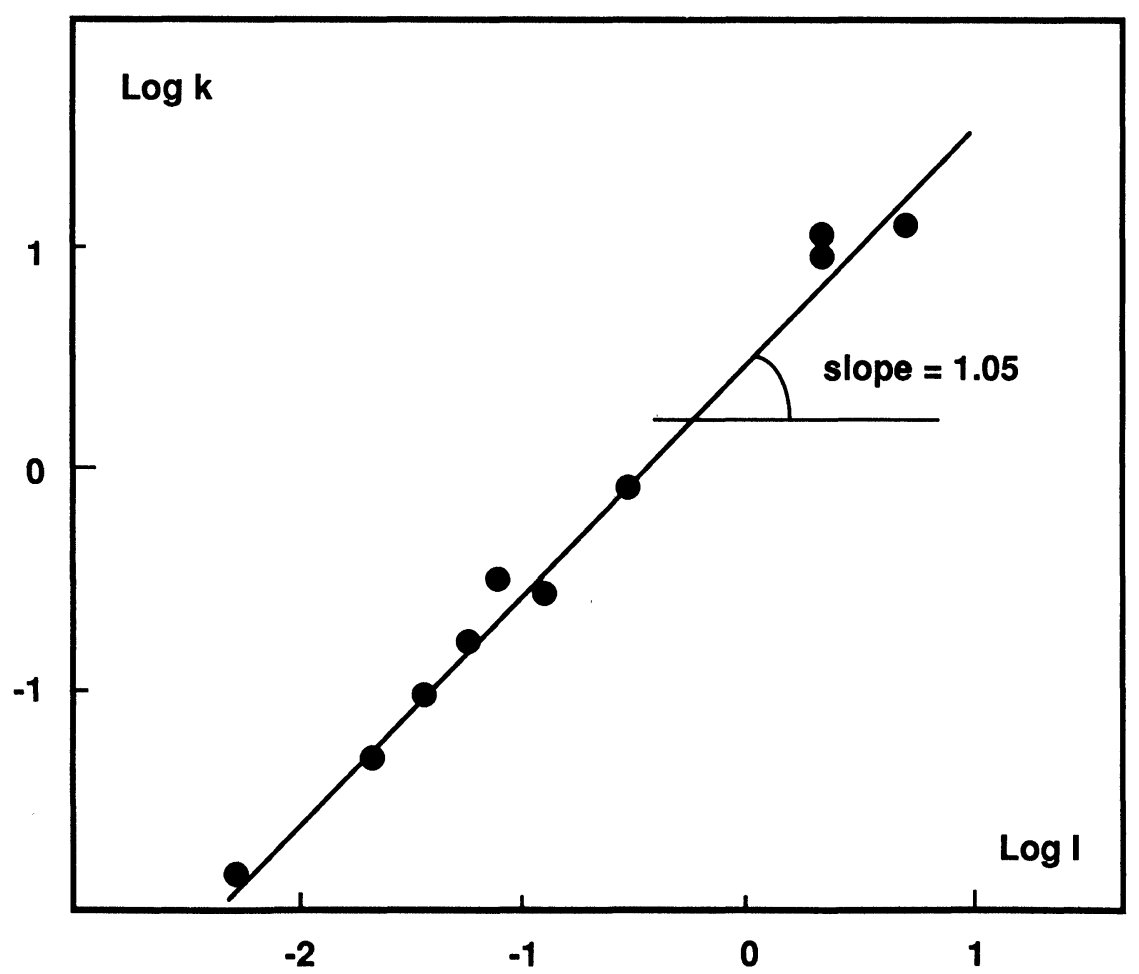

Figure 6 Linear dependence of $\log k$ against $\log$ i. 


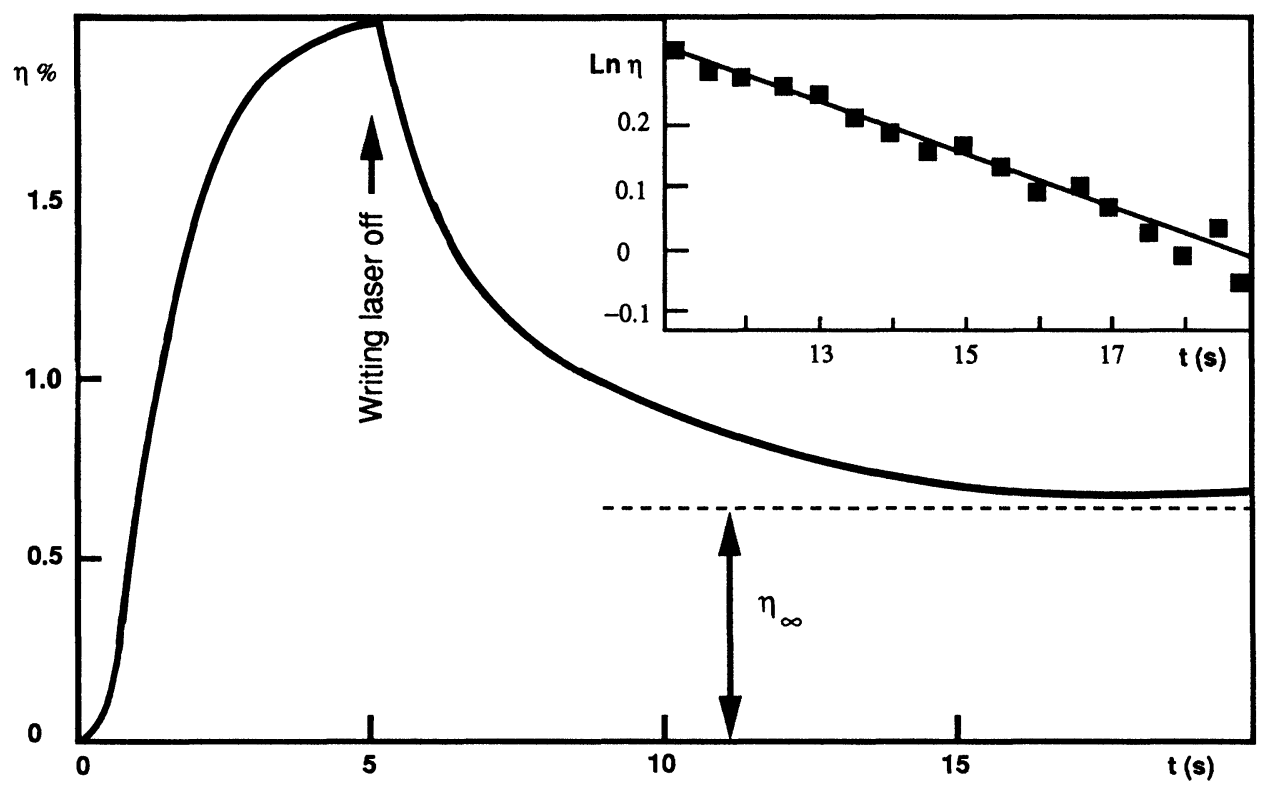

Figure 7 Building up and decay of the record with Methyl Orange in PVA; $I=3.5 \mathrm{~mW} / \mathrm{cm}^{2}$; time of illumination $=5 \mathrm{~s}$.

$\left(\right.$ at $\left.20^{\circ} \mathrm{C}\right)$. According to the theory developed by Braüchle and coworkers, the lifetime of the transient species responsible of the index modulation is twice that of the grating itself ${ }^{26}$ i.e., 10 seconds in the experiments described in this paper. This result is in line with those reported by Wang et al. ${ }^{41}$ in polymethylmethacrylate and polystyrene.

Table 2 shows the sensitivity of this thermal back process to acidity; as no $\mathrm{pH}$ value is available in a solid medium, this table merely reports on the influence of adding increasing amounts of polyacrylic acid in the polymer matrix on the lifetime of the cis isomer. Unfortunately, the poor time-resolution of this technique does not allow to reach the upper limit of the corresponding decay rate constant (on increasing acidity). Nevertheless, it gives a direct evidence of the catalyzing effect of $\mathrm{H}^{+}$in a polymer matrix such as PVA; it also suggests that in this environment, the back process to the trans form does not involve such a broad twist of the molecule but rather a concerted buckling of the bonds around the nitrogen center i.e., an isomerization by inversion. This fundamental question which is still under investigation and debate will form the subject of a forthcoming paper. ${ }^{42}$

Table 2 Lifetime of the cis species of Methyl Orange as a function of the acidity introducted by polyacrylic acid in PVA

\begin{tabular}{lccccc}
\hline$\%$ PAA in PVA & 0 & 0.1 & 0.2 & 0.5 & 1 \\
\hline$\tau_{\text {cis }}\left(\right.$ at $\left.20^{\circ} \mathrm{C}\right)$ & 10 & 1.05 & 0.21 & $0.012<5 \mathrm{~ms}$
\end{tabular}




\section{LASER-INDUCED PHOTOPOLYMERIZATION}

Several reports on polymerizations induced by UV laser beams have been published within the last few years, ${ }^{43-55}$ most of them dealing with multifunctional monomers which were found to polymerize almost instantly under such extreme conditions, leading to a polymer network of very high crosslink density. The process that transforms a liquid monomer into a solid insoluble material is usually called curing. Under intense laser irradiation, the photo-curing occurs within a fraction of a second, which explains why little attention has been directed so far toward a quantitative investigation of the kinetics of such ultrafast reactions. The extent of the polymerization can be followed continuously by monitoring the heat evolved, using a photodifferential calorimeter. However, owing to its relatively long response time, this technique ${ }^{46,55,56,57}$ proved only able to analyse correctly the kinetics of polymerizations which proceed in more than $10 \mathrm{~s}$, thus requiring to lower considerably the laser fluence rate. The same limitation is to be found in the other time-resolved techniques, like dilatometry ${ }^{58}$ or infrared radiometry. ${ }^{59}$ By contrast, laserinterferometry ${ }^{54}$ permits to study ultrafast polymerizations by monitoring in real time the diffraction efficiency of the grating formed upon laser irradiation. This elegant method still gives only qualitative information about the polymerization kinetics and provides no evaluation of the actual reaction rate or degree of polymerization.

The kinetics of laser-induced polymerizations can also be determined by monitoring the disappearance of the IR absorption, characteristic of the polymerizable double bond. This analytical method has proved extremely valuable for measuring polymerizable rates and quantum yields of reactions that develop in the millisecond time scale ${ }^{60-61}$ Figure 8 shows the kinetic profile obtained by plotting the degree of conversion against the irradiation time for a diacrylate photoresist exposed to the $363.8 \mathrm{~nm}$ emission of a $\mathrm{CW}$ argon ion laser. In the presence of air or pure oxygen, typical $S$ shape kinetic curves were obtained. The induction period observed in the early stages is primarily due to the well-known inhibition effect of oxygen on those radical-induced polymerizations. ${ }^{62}$ It disappeared almost completely when the laser irradiation was carried out under a nitrogen purge, an exposure as short as 20 milliseconds being sufficient to obtain a totally insoluble polymer. The progressive slowing down observed at degrees of conversion above $40 \%$ is the direct consequence of the network formation and the subsequent gelification which reduces the segmental mobility of the growing polymer chains. It thus remains in the final polymer a certain amount of unreacted monomer, 10 to $30 \%$, depending on the chemical structure and functionality of the monomer used. ESR investigations ${ }^{63}$ and posteffect experiments ${ }^{64}$ have recently confirmed the presence of long-living radicals in UV-cured multiacrylic monomers. From the reaction profile of Figure 8, the rate of polymerization $\left(R_{p}\right)$ can be determined at any moment by taking the slope of the conversion versus time curve. Measuring the laser fluence rate $\left(I_{0}\right)$ at the sample position and the absorbance of the sample at the laser wavelength (A), allows the overall polymerization quantum yield $\left(\Phi_{p}\right)$ to be calculated from the following equation: $\Phi_{p}=R_{p} / I_{0}\left(1-\mathrm{e}^{-2.3 \mathrm{~A}}\right)$. For multiacrylic monomers, each laser photon 
was found to induce the polymerization of 2000 monomer units for films in contact with air, and up to 10,000 in the absence of oxygen, ${ }^{45,65}$ thus indicating how efficiently the chain reaction develops in such systems. Similar results were obtained by using pulsed lasers that emit in the UV range, at $337.1 \mathrm{~nm}$ for the nitrogen laser or at $308 \mathrm{~nm}$ for the $\mathrm{XeCl}$ laser. With the most reactive system, a single laser shot, $8 \mathrm{~ns}$ wide and of $5 \mathrm{~mJ}$ energy, proved to be sufficient to get a $100 \%$ insoluble polymer..$^{45}$

One of the major disadvantages of IR spectroscopy is that it is a time-consuming technique which requires tedious point by point measurements. Furthermore, it integrates into the final result the postpolymerization effect which develops during the few second time lapse between the end of the UV exposure and the actual measurement. These difficulties can be overcome by using real time infrared (RTIR) spectroscopy in order to follow continuously and in real time polymerizations induced by $\mathrm{CW}$ or pulsed laser beams. By exposing the monomer sample simultaneously to the laser beam and to the analyzing IR beam, one can directly monitor on a transient memory recorder the drop in the IR absorbance of the reactive function due to the polymerization. ${ }^{66,67}$ Figure 9 shows typical polymerization profiles recorded for an acrylic monomer, exposed to the $337.4 \mathrm{~nm}$ emission of a $\mathrm{Kr}^{+}$ laser, in the presence of various photoinitiators. Since the absorbance at $812 \mathrm{~cm}^{-1}$ is directly related to the number of acrylate double bonds which have polymerized, the curve recorded actually represents the variation of the degree of conversion with the laser exposure time. A single experiment, which lasts a fraction of a second, is thus sufficient to evaluate the important kinetic parameters (induction period, rate and quantum yield of polymerization, photosensitivity) and assess the performance of new photoinitiators and monomers. The great sensitivity of RTIR spectroscopy

Conversion (\%)

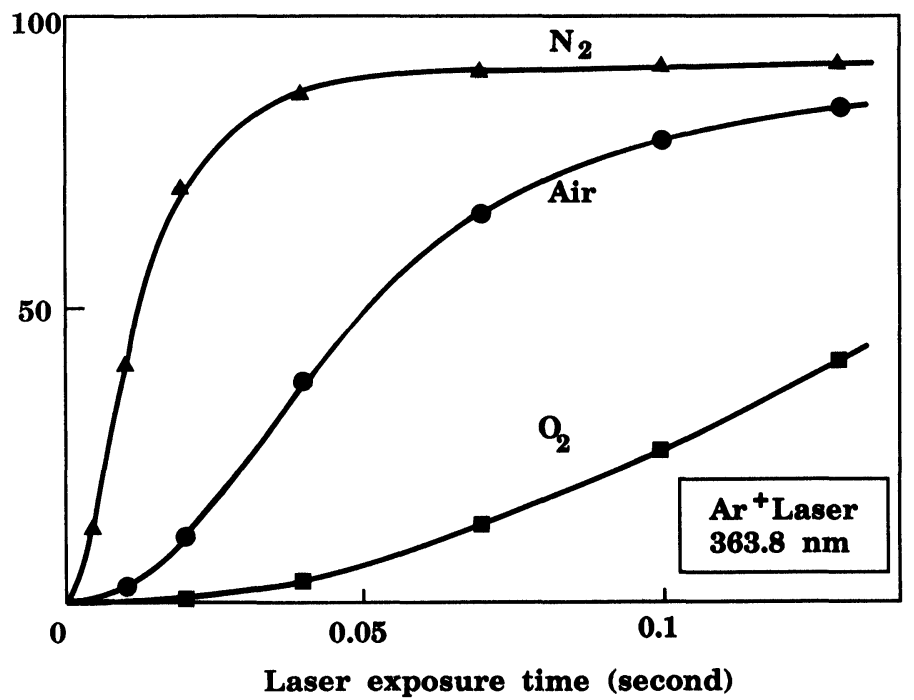

Figure 8 Influence of oxygen on the laser-induced polymerization of a diacrylate monomer, studied by infrared spectroscopy. 


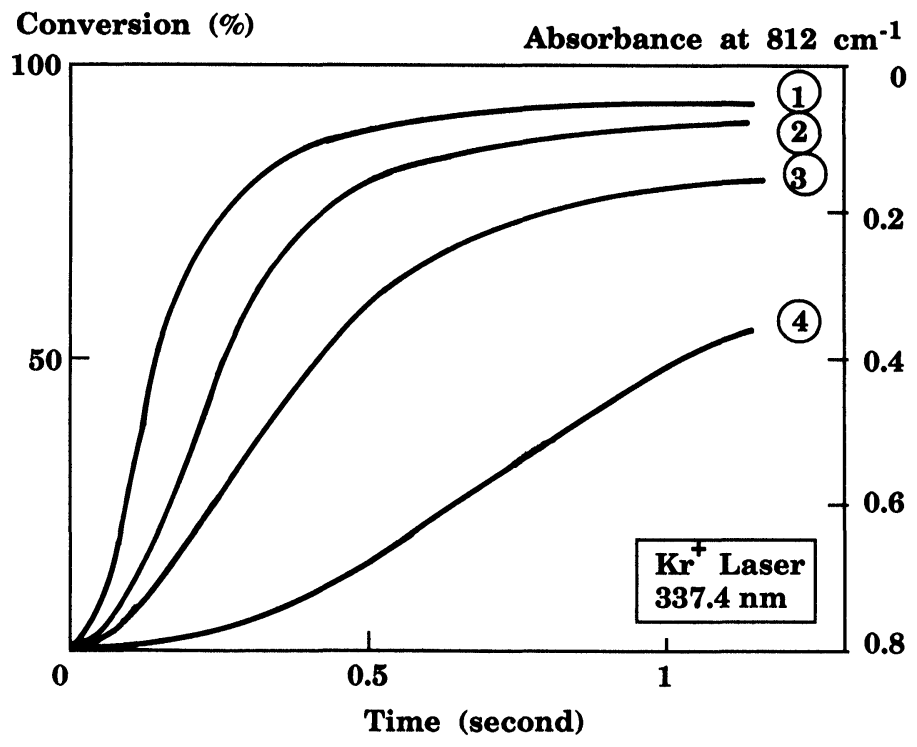

Figure 9 Influence of the photoinitiator $(5 \%)$ on the laser-induced polymerization of a diacrylate monomer. Kinetic profiles recorded by time-resolved infrared photo-spectroscopy (RTIR); Fluence rate: $200 \mathrm{~mW} \mathrm{~cm}^{-2}$.

1: 2 benzyl-2-dimethylamino-1-(4 morpholinophenyl) butanone 1

2: 2,2 dimethoxy, phenylacetophenone

3: 1 . benzoylcyclohexanol

4: benzophenone + methyl diethanolamine.

allows chemical changes as small as $1 \%$ to be detected in a $1 \mu \mathrm{m}$ thick film, a performance unchallenged so far by any alternative method. The influence of such important factors as the radiation wavelength, fluence rate, film thickness, photoinitiator concentration etc. have thus been evaluated quantitatively for various photopolymerizable systems. ${ }^{65-66}$ Another advantage of this technique is that the rate constants of propagation $\left(k_{p}\right)$ and of termination $\left(k_{t}\right)$ can be directly evaluated from the polymerization kinetic curve recorded upon laser irradiation and in the dark, for any monomer system. ${ }^{68}$ Furthermore, a study of the very early stages of the polymerization allowed both the growth profile of the radical concentration to be obtained and the exposure time needed to reach steady-state conditions to be determined, for highly reactive laser-curable acrylic systems. ${ }^{65}$

Laser-induced photopolymerization can be greatly accelerated, either by increasing the power output, or by finely focusing the laser beam..$^{53}$ Complex patterns can thus be written at micronic resolution onto the photo-responsive polymer by means of a computer-directed laser beam, much in the same way as in electron beam lithography. This direct maskless writing of polymer relief images is expected to find its main applications in microelectronics for the production of high-resolution semi-conductor devices and in the graphic arts. Other possible uses of intense laser irradiation include three-dimension modelling, the curing of thick sections of polymers ${ }^{54}$ or of composites and the high speed treatment of optical fibers or microcircuits ${ }^{69}$ by UV curable coatings. 


\section{CONCLUSION}

This survey is aimed at taking stock of the recent developments of laser techniques for the study of photo-induced polymerizations and all kinds of photoprocesses taking place in polymer matrixes.

The first part which is devoted to time-resolved absorption-spectroscopy shows the decisive importance of this technique for the kinetic and mechanistic study of the photoinitiation process in model systems. Any prospective investigation of new classes of potentially active photoinitiator should be based on an approach involving this technique. The interest of holographic spectroscopy lies in its perfect suitability for time-resolved studies in polymer films. Any photo-induced process which goes along with the weakest refractive index change can be investigated by this technique: isomerization, diffusion, polymerization. Time-resolved infrared spectroscopy has proved to be a well-suited technique for studying in real time and quantitatively such ultrafast polymerizations induced by laser irradiation. The important kinetic parameters can be determined instantly from the conversion versus time profiles, which are directly recorded in the millisecond timescale. Laser-cured polymers were shown to exhibit outstanding properties, which makes them particularly well-suited for applications where very large speed, high resolution and great penetration are required, such as for the ultrafast coating of materials, the writing of micronic relief patterns and the 3D creation of solid objects.

\section{References}

1. J. Faure et J. Joussot-Dubien, Images de la Chimie 1977, p. 14.

2. J. Faure, J. P. Fouassier, D. J. Lougnot et R. Salvin, Nouv. J. Chim. 1, 15 (1977).

3. C. G. Roffey, Photopolymerization of Surface Coatings (J. Wiley Sons, New York, 1982).

4. UV-Curing: Science and Technology, S. P. Pappas Ed. (Technology Marketing Publication, Stamford, 1978).

5. Lasers in Polymer Science and Technology Applications, J. P. Fouassier and J. F. Rabek Eds. (CRC Press Inc., Boca Raton, USA, 1989).

6. J. F. Rabek, Mechanisms of Photophysical and Photochemical Reactions in Polymer: Theory and Practical Applications (Wiley, New York, 1987).

7. J. P. Fouassier, D. J. Lougnot, A. Payerne and F. Wieder, Chem. Phys. Lett. 135, 30 (1987).

8. J. P. Fouassier and A. Merlin, J. Photochem. 12, 17 (1980).

9. D. J. Lougnot, P. Jacques and J. P. Fouassier, J. Photochem. 19, 59 (1982).

10. J. P. Fouassier and D. J. Lougnot, J. Chem. Soc., Faraday Trans. 1, 83, 2935 (1987).

11. J. P. Fouassier, D. J. Lougnot and J. C. Scaiano, Chem. Phys. Lett. 160(3), 335 (1989).

12. D. J. Lougnot, C. Turck and J. P. Fouassier, Macromolecules 22, 108 (1989).

13. J. P. Fouassier, D. J. Lougnot, G. Li Bassi and C. Nicora, Polymer 30, 245 (1989).

14. W. Schnabel, T. Sumiyoshi, in New Trends in the Photochemistry of Polymer (N. S. Allen and J. F. Rabek Eds., Elsevier Publ., London, 1985).

15. D. J. Lougnot and J. P. Fouassier, J. Polym. Sci., Part A: Polym. Chem. 26, 1021 (1988).

16. J. P. Fouassier and D. J. Lougnot, J. Appl. Polym. Sci. 34, 477 (1987).

17. J. P. Fouassier, P. Jacques and M. V. Encinas, Chem. Phys. Lett. 148, 309 (1988).

18. R. J. De Voe, M. R. V. Sahyun, E. Smidt, N. Serpone and D. K. Sharma, Can. J. Chem. 66, 319 (1988).

19. J. P. Fouassier, D. Burr and J. V. Crivello, J. Photochem. 49, 317 (1989).

20. J. V. Crivello, Advances in Polymer Science 62 (T. Saegusa Ed., Springer Verlag, Berlin, 1984).

21. A. Roloff, K. Meier and M. Riediker, Pure and Appl. Chem. 58, 1267 (1986).

22. J. P. Fouassier, E. Chesneau and M. Le Baccon, Makromol. Chem., Macromol. Symp. 9, 223 (1988). 
23. W. Rutsch, G. Berner, R. Kirchmayer, R. Husler, G. Rist, N. Buhler, Proc. Radcure, Atlanta 1984 (SME Ed., Dearborn, USA Techn. Paper FC 84 252).

24. W. J. Tomlison, E. A. Chandross, Adv. Photochem. 12, 201 (1980).

25. J. Feinberg, SPIE 532, 119 (1985).

26. C. Braüche and D. M. Burland, Angew. Chem., Int. Ed. Engl. 22, 582 (1983) and references cited therein.

27. F. W. Deeg, J. Pinsl and C. Braüchle, J. Phys. Chem. 90, 5710 (1986).

28. J. Pinsl, F. W. Deeg and C. Braüchle, Appl. Phys. B 40, 77 (1988).

29. F. W. Deeg, J. Pinsl and C. Braüchle, IEEE J. Quantum Electron, QE-22, 1476 (1986).

30. J. Pinsl, M. Gehrtz and C. Braüchle, J. Phys. Chem. 90, 6754 (1986).

31. D. M. Burland, Acc. Chem. Res. 16, 218 (1983).

32. D. M. Burland, IEEE J. Quantum Electron. QE-22, 1469 (1986).

33. A. C. Testa and U. P. Wild, Phys. Chem. 90, 4302 (1986).

34. D. G. Milles, P. D. Lamb, K. W. Rhee and C. S. Johnson, J. Phys. Chem. 87, 4815 (1983).

35. H. Kogelnik, Bell. Syst. Tech. J. 48, 2909 (1969).

36. R. J. Collier, C. B. Burckard and L. H. Lin, Optical Holography (Academic, New York, 1971).

37. G. Champetier, R. Buvet, J. Neel and P. Sigwalt, Chimie Macromoléculaire; (Hermann, Paris, 1970), p. 82.

38. C. Carré, D. J. Lougnot and J. P. Fouassier, Macromolecules 22791 (1989).

39. P. Jacques, J. Chim. Phys. 79, 4, 351 (1982).

P. Jacques, Dyes and Pigments 9, 129 (1988).

40. J. A. Couture, C. Carré, D. J. Lougnot and P. Jacques, to be published.

41. S. S. Goug, D. Christensen, J. Zhang and C. H. Wang, J. Phys. Chem. 91, 4504 (1987).

42. D. J. Lougnot and C. Carré, to be published.

43. O. F. Olaji, I. Bitai and G. Gleixner, Makromol. Chem. 186, 2569 (1985).

44. O. F. Olaji, I. Bitai, Makromol. Chem. Rapid Commun. 9, 275 (1988).

45. C. Decker, ACS Sym. Ser. 266, 207 (1984).

46. C. E. Hoyle, R. D. Hensel and M. B. Grubb, Polym. Photochem. 4, 69 (1984).

47. C. Decker, Microcircuit Eng. 82, 299 (1982).

48. C. Decker, SME Techn. Paper FC. 83, 265 (1983).

49. C. Decker, Polym. Photochem. 3, 131 (1983).

50. J. Y. Tsao and D. J. Ehrlich, Appl. Phys. Lett. 42, 997 (1983).

51. C. Decker, Polym. Mater. Sci. Eng. 49, 32 (1983).

52. C. Decker, J. Polym. Sci., Polym. Chem. Ed. 21, 2451 (1983).

53. C. Decker, J. Coat. Techn. 56, 29 (1984).

54. C. Decker, in Radiation Curing of Polymers, D. R. Randell Ed., Roy. Sci. Chem. London 1986, 6.

55. C. E. Hoyle, M. Trapp and C. H. Chang, Polym. Mater. Sci. Eng. 57, 579 (1987).

56. X. T. Phan and M. B. Grubb., J. Macromol. Sci. Chem. A 25, 143 (1988).

57. C. E. Hoyle, R. D. Hensal and M. B. Grubb, J. Polym. Sci. Polym. Chem. Ed. 22, 1865 (1984).

58. D. R. Pemberton, A. F. Johnson, Polymer 25, 529 (1984).

59. G. B. Tanny, A. Lubelsky, Z. Rav-Noy and E. Shchon, S.M.E. Techn. Paper FC 85-440 (1985).

60. C. Decker and T. Bendaikha, Europ. Polym. J. 20, 753 (1983).

61. C. Decker, J. Coat. Techn. 59 (751), 97 (1987).

62. C. Decker and A. Jenkins, Macromolecules 18, 1241 (1985).

63. C. Decker and K. Moussa, J. Polym. Sci., Polym. Chem. Ed. 25, 739 (1987).

64. C. Decker and K. Moussa, J. Appl. Polym. Sci. 34, 1603 (1987).

65. C. Decker and K. Moussa, Macromolecules 22, 4455, (1989).

66. C. Decker and K. Moussa, Macromol. Chem. 189, 2381 (1988).

67. C. Decker and K. Moussa, Polym. Mater. Sci. Eng. 60, 547 (1989).

68. C. Decker and K. Moussa, Europ. Polym. J. (in press).

69. C. Decker, Proc. Symp. on Polymers for Microelectronics, Tokoyo 1989. 